\title{
Preparações radiofarmacêuticas e suas aplicações
}

\author{
Rita Oliveiraa ${ }^{1,2}$, Delfim Santos ${ }^{2}$, Domingos Ferreira ${ }^{2}$, Pedro Coelho ${ }^{1,2}$, Francisco Veiga $^{3 *}$ \\ ${ }^{1}$ Faculdade de Ciências da Saúde da Universidade Fernando Pessoa, ${ }^{2}$ Faculdade da Farmácia da Universidade do \\ Porto, ${ }^{3}$ Faculdade de Farmácia da Universidade de Coimbra
}

*Correspondência:

F. Veiga

Faculdade de Farmácia Universidade de Coimbra 3000-295 Coimbra, Portugal E-mail: fveiga@ci.uc.pt
Os radiofármacos são compostos, sem ação farmacológica, que têm na sua composição um radionuclídeo e são utilizados em Medicina Nuclear para diagnóstico e terapia de várias doenças. Para aplicações de diagnóstico em Medicina Nuclear utilizam-se radiofármacos que apresentam na sua constituição radionuclídeos emissores de radiação $\gamma$ ou emissores de pósitrons $\left(\beta^{+}\right)$, já que o decaimento destes radionuclídeos dá origem a radiação eletromagnética penetrante, que consegue atravessar os tecidos e pode ser detectada externamente. Os radiofármacos para terapia devem incluir na sua composição um radionuclídeo emissor de partículas ionizantes ( $\alpha, \beta$ ou elétrons Auger), pois a sua ação se baseia na destruição seletiva de tecidos. Existem dois métodos tomográficos para aquisição de imagens em Medicina Nuclear: o SPECT (Tomografia Computarizada de Emissão de Fóton Único), que utiliza radionuclídeos emissores $\gamma\left({ }^{99 m} \mathrm{Tc},{ }^{123} \mathrm{I},{ }^{67} \mathrm{Ga},{ }^{201} \mathrm{Tl}\right)$ e o PET (Tomografia por Emissão de Pósitrons), que usa radionuclídeos emissores de pósitrons $\left({ }^{11} \mathrm{C},{ }^{13} \mathrm{~N},{ }^{15} \mathrm{O},{ }^{18} \mathrm{~F}\right)$. Os radiofármacos podem ser classificados em radiofármacos de perfusão (ou $1^{a}$ geração) e radiofármacos especificos (ou $2^{a}$ geração). Os radiofármacos de perfusão são transportados no sangue e atingem o órgão alvo na proporção do fluxo sanguíneo. Os radiofármacos ditos específicos contêm molécula biologicamente ativa, que se liga a receptores celulares e que deve manter a sua bioespecificidade mesmo após ligação ao radionuclídeo. Assim, nestes radiofármacos, a fixação em tecidos ou órgãos é determinada pela capacidade da biomolécula de reconhecer receptores presentes nessas estruturas biológicas. As preparações radiofarmacêuticas são obtidas prontas para uso, em kits frios ou em preparações autólogas. De acordo com o tipo de preparação, existe um processo de controle de qualidade próprio. A maior parte dos radiofármacos em uso clínico corresponde a agentes de perfusão. Atualmente, o esforço de investigação na área da química radiofarmacêutica centra-se no desenvolvimento de radiofármacos especificos que possam fornecer informação, ao nivel molecular, relativa às alterações bioquímicas associadas às diferentes patologias.
Unitermos

- Radiofármaco

- Radionuclídeo

- Diagnóstico

- Terapêutica 


\section{INTRODUÇÃO}

Os radiofármacos são compostos, sem ação farmacológica, que têm na sua composição um radionuclídeo, e são utilizados em Medicina Nuclear para diagnóstico e terapia de várias doenças (European Pharmacopeia, 2005). As características físico-químicas do radiofármaco determinam a sua farmacocinética, isto é, a sua fixação no órgão alvo, metabolização e eliminação do organismo, enquanto que as características físicas do radionuclídeo determinam a aplicação do composto em diagnóstico ou terapia.

Além das aplicações em Medicina Nuclear, a radioatividade tem sido aplicada em Medicina sob diferentes formas:

- Fonte de radiação externa ao organismo, em radiologia e radioterapia convencional;

- Radioesterilização de produtos e materiais com utilização médica;

- Doseamento de hormônios.

A Medicina Nuclear obtém as imagens através da administração de radiofármacos e medindo externamente a radiação emitida que atravessa o organismo, ao contrário das técnicas radiológicas convencionais, que medem a absorção da radiação aplicada externamente. A dose de um radiofármaco necessária a um exame é muito mais baixa do que a dose de agentes de contraste utilizada em outras técnicas de diagnóstico, tais como a radiografia e a ressonância magnética nuclear (RMN). Assim, em Medicina Nuclear não ocorrem efeitos farmacológicos, sendo uma técnica não invasiva que permite avaliar a função e não só a morfologia do órgão. Do ponto de vista do paciente, as técnicas são simples e apenas requerem administração endovenosa, oral ou inalatória de um radiofármaco e as reações adversas são excepcionais (Dilworth et al., 1998).

A radiografia é uma técnica de diagnóstico em que se registra a permeabilidade dos tecidos aos raios X. Utilizamse muitas vezes agentes de contraste, que promovem a absorção dos raios $\mathrm{X}$ aumentando o contraste das imagens (Clarke et al., 1999; Guo et al., 1999; Elder et al., 1994).

A RMN é uma técnica tomográfica, que utiliza os núcleos dos prótons das moléculas de água existentes nos tecidos como sondas magnéticas naturais. Quando é aplicado um campo magnético, os núcleos absorvem diferentes radiofreqüências conforme a orientação que assumiram após aplicação do campo magnético. Esta informação é convertida num registro que dá a posição espacial dos núcleos, isto é, uma imagem. O contraste observado nestas imagens deve-se ao fato de diferentes tecidos terem diferentes quantidades de moléculas de água e ainda pelo fato dos núcleos possuírem graus de mobilidade diferentes.

Para que a qualidade das imagens de raios $\mathrm{X}$ e de RMN ofereçam contraste mais elevado, utilizam-se agen- tes de contraste que são administrados em doses elevadas, o que pode acarretar problemas alérgicos ou de toxicidade. Pelo contrário, a massa de produto que se introduz no organismo quando se utiliza um radiofármaco, em Medicina Nuclear, é mínima, não se provocando, em geral, qualquer problema de alergia ou toxicidade (Clarke et al., 1999).

Apesar das técnicas de RMN e de raios X apresentarem melhor resolução, estas técnicas são menos específicas. As técnicas de Medicina Nuclear fornecem imagens de menor detalhe anatômico, mas permitem avaliação funcional (Clarke et al., 1999; Dilworth et al., 1998). Todavia, para determinados diagnósticos, estas técnicas podem considerar-se complementares.

Em termos de terapia, a Medicina Nuclear utiliza radiofármacos, que têm na sua composição um radionuclídeo, que emite radiação ionizante. O efeito desta radiação sobre os tecidos ou órgãos alvo promove a destruição das células tumorais. A captação do radiofármaco no órgão alvo deve ser seletiva, de modo a minimizar os efeitos secundários, que são uma das grandes desvantagens da radioterapia externa em que é delicado controlar a dose de radiação fornecida, especialmente para tratamento de metástases disseminadas. No caso da radioterapia externa, os tecidos saudáveis estão também expostos a elevadas doses de radiação, o que pode aumentar a incidência de leucemias e cancros secundários (Elder et al., 1994; Volkert et al., 1999).

\section{BREVES NOÇÕES DE RADIOATIVIDADE}

Um nuclídeo é uma espécie caracterizada pelo seu número atômico $(\mathrm{Z})$ e número de massa $(\mathrm{A})$, cuja estabilidade é determinada pela relação entre o número de prótons e neutrons.

Sempre que um núcleo é instável (radionuclídeo), transforma-se espontaneamente noutro mais estável emitindo partículas ( $\alpha, \beta, \beta^{+}$, elétrons Auger) e/ou radiação eletromagnética (raios $\gamma$ ou X). Este fenômeno denomina-se radioatividade e a sua unidade de medida designa-se por atividade, que é o número de desintegrações por segundo (d.p.s., Ci ou Bq).

O tempo de meia-vida $\left(\mathrm{t}_{1 / 2}\right)$ é definido como o tempo necessário para reduzir à metade a atividade inicial de um radionuclídeo, sendo independente das condições fisico-químicas e característico de cada radionuclídeo.

Apesar das radiações $\beta$ e $\gamma$ terem poder penetrante maior dos que as partículas $\alpha$, são as que originam menores danos biológicos. Estes danos biológicos potencializam-se com a ionização em meios aquosos (como é o caso do corpo humano), que pode originar a quebra das moléculas de água e a formação de radicais livres, que podem danificar o material biológico. 


\section{PRODUÇÃO DE RADIONUCLÍDEOS}

Os radionuclídeos usados em Medicina Nuclear para diagnóstico e terapia são produzidos artificialmente em reatores ou aceleradores de partículas. Podem, ainda, ser acessíveis através de geradores de radioisótopos, que permitem a utilização de radionuclídeos de $\mathrm{t}_{1 / 2}$ curto, a partir do decaimento de um radionuclídeo com $\mathrm{t}_{1 / 2}$ longo. Estes radionuclídeos de $\mathrm{t}_{1 / 2}$ longo são produzidos em reator ou cíclotron (Saha, 1998).

Os radionuclídeos que decaem por emissão de partículas $\beta^{-}$são geralmente produzidos em reator (Saha, 1998), por fissão do ${ }^{235} \mathrm{U}$ ou por reações de captura de neutrons (n, $\gamma$ ou n,p) numa amostra alvo apropriada. Os radionuclídeos que decaem por captura eletrônica ou emissão de partículas $\beta^{+}$são produzidos em cíclotrons (Saha, 1998). Nestas reações, partículas de elevada energia interagem com núcleos estáveis de alvos apropriados, originando produtos deficientes em prótons. Neste processo, as partículas que interagem com as amostras alvo podem ser prótons, dêuterons, partículas $\alpha$ ou ${ }^{3} \mathrm{He}$.

A Tabela I resume os métodos de produção dos radionuclídeos mais utilizados (Saha, 1998; Vallabhajosula, 2001).

Os geradores permitem obter um radionuclídeo de $t_{1 / 2}$ curto a partir de um radionuclídeo de $\mathrm{t}_{1 / 2}$ longo. As propriedades químicas dos dois radionuclídeos têm que ser distintas para que sejam facilmente separados (Saha, 1998).

Os geradores são constituídos por uma coluna de alumina, ou por uma resina de troca iônica, na qual se fixa o radionuclídeo "pai" de tempo de meia-vida longo. Por decaimento deste último, forma-se o radionuclídeo "filho", que é separado por eluição, com um eluente adequado. O eluído pode ser utilizado diretamente em aplicações clínicas, constituindo, neste caso, a substância radiofarmacêutica, ou pode servir para preparar radiofármacos mais complexos. O eluído deve ser obtido na forma estéril e isenta de pirogênios. A utilização do gerador deve ser feita de forma a nunca se perder a esterilidade e a apirogenicidade. Na Figura 1 apresenta-se esquematicamente o gerador ${ }^{99} \mathrm{Mo} /{ }^{99 \mathrm{~m}} \mathrm{Tc}$.

No caso do gerador ${ }^{99} \mathrm{Mo} /{ }^{99 \mathrm{~m}} \mathrm{Tc}$, a atividade do radionuclídeo "fillho" ${ }^{99 \mathrm{~m}} \mathrm{Tc}$ ) vai aumentando à medida que o radionuclídeo "pai" $\left({ }^{99} \mathrm{Mo}\right)$ vai decaindo. $\mathrm{O}{ }^{99} \mathrm{Mo}$, na forma química de $\mathrm{MoO}_{4}^{2-}$, encontra-se adsorvido numa coluna de alumina e por eluição com soro fisiológico é apenas eluído o ${ }^{99 \mathrm{~m}} \mathrm{TcO}_{4}^{-}$, recolhido sob vácuo, enquanto o molibdato fica retido na coluna (Figura 1).

Um gerador ideal deverá ter uma proteção de chumbo para minimizar a exposição à radiação do experimentador, deverá ser simples, rápido de utilizar e originar
TABELA I - Métodos de produção e/ou obtenção de radionuclídeos

\begin{tabular}{|c|c|c|}
\hline Fonte & Radionuclídeo & Reação nuclear \\
\hline Reator & $\begin{array}{l}{ }^{32} \mathrm{P} \\
{ }^{67} \mathrm{Cu} \\
{ }^{177} \mathrm{Lu} \\
{ }^{89} \mathrm{Sr} \\
{ }^{186} \mathrm{Re} \\
{ }^{153} \mathrm{Sm} \\
{ }^{99} \mathrm{Mo}\end{array}$ & $\begin{array}{l}{ }^{235} \mathrm{U}(\mathrm{n}, \mathrm{f}){ }^{131} \mathrm{I} \\
{ }^{130} \mathrm{Te}(\mathrm{n}, \gamma){ }^{131} \mathrm{Te}^{\mathrm{b}-} \rightarrow{ }^{131} \mathrm{I} \\
{ }^{31} \mathrm{P}(\mathrm{n}, \gamma){ }^{32} \mathrm{P} /{ }^{32} \mathrm{~S}(\mathrm{n} . \mathrm{p})^{32} \mathrm{P} \\
{ }^{67} \mathrm{Zn}(\mathrm{n}, \mathrm{p}){ }^{67} \mathrm{Cu} \\
{ }^{176} \mathrm{Lu}(\mathrm{n}, \gamma){ }^{177} \mathrm{Lu} \\
{ }^{88} \mathrm{Sr}(\mathrm{n}, \gamma){ }^{89} \mathrm{Sr} \\
{ }^{185} \mathrm{Re}(\mathrm{n}, \gamma){ }^{186} \mathrm{Re} \\
\left.{ }^{152} \mathrm{Sm}(\mathrm{n}, \gamma)\right)^{153} \mathrm{Sm} \\
{ }^{235} \mathrm{U}(\mathrm{n}, \mathrm{f}){ }^{99} \mathrm{Mo} / \\
{ }^{98} \mathrm{Mo}(\mathrm{n}, \gamma){ }^{99} \mathrm{Mo}\end{array}$ \\
\hline $\begin{array}{l}\text { Acelerador/ } \\
\text { cíclotron }\end{array}$ & $\begin{array}{l}{ }^{123} \mathrm{I} \\
{ }^{67} \mathrm{Ga} \\
{ }^{111} \mathrm{In} \\
{ }^{201} \mathrm{Tl} \\
{ }^{11} \mathrm{C} \\
{ }^{13} \mathrm{~N} \\
{ }^{15} \mathrm{O} \\
{ }^{18} \mathrm{~F} \\
{ }^{124} \mathrm{I} \\
{ }^{211} \mathrm{At} \\
{ }^{64} \mathrm{Cu}\end{array}$ & $\begin{array}{l}{ }^{121} \mathrm{Sb}(\alpha, 2 \mathrm{n}){ }^{123} \mathrm{I} \\
{ }^{68} \mathrm{Zn}(\mathrm{p}, 2 \mathrm{n}){ }^{67} \mathrm{Ga} \\
{ }^{111} \mathrm{Cd}(\mathrm{p}, \mathrm{n}){ }^{111} \mathrm{In} \\
\left.{ }^{203} \mathrm{Tl}(\mathrm{p}, 3 \mathrm{n})\right)^{201} \mathrm{~Pb} \rightarrow{ }^{201} \mathrm{Tl} \\
\left.{ }^{14} \mathrm{~N}(\mathrm{p}, \alpha)\right)^{11} \mathrm{C} \\
\left.\left.{ }^{16} \mathrm{O}(\mathrm{p}, \alpha)\right)^{13} \mathrm{~N} /{ }^{13} \mathrm{C}(\mathrm{p}, \mathrm{n})\right)^{13} \mathrm{~N} \\
{ }^{14} \mathrm{~N}(\mathrm{~d}, \mathrm{n}){ }^{150} /{ }^{15} \mathrm{~N}(\mathrm{p}, \mathrm{n}){ }^{15} \mathrm{O} \\
{ }^{18} \mathrm{O}(\mathrm{p}, \mathrm{n}){ }^{18} \mathrm{~F} \\
{ }^{124} \mathrm{Te}(\mathrm{d}, 2 \mathrm{n}){ }^{124} \mathrm{I} \\
{ }^{207} \mathrm{Bi}(\alpha, 2 \mathrm{n}){ }^{211} \mathrm{At} \\
{ }^{64} \mathrm{Ni}(\mathrm{p}, \mathrm{n}){ }^{64} \mathrm{Cu}\end{array}$ \\
\hline Gerador & $\begin{array}{l}{ }^{99 \mathrm{~m}} \mathrm{Tc} \\
{ }^{68} \mathrm{Ga} \\
{ }^{90} \mathrm{Y} \\
{ }^{188} \mathrm{Re} \\
{ }^{212} \mathrm{Bi}\end{array}$ & $\begin{array}{l}{ }^{99} \mathrm{Mo}^{\beta-} \rightarrow{ }^{99 \mathrm{~m}} \mathrm{Tc} \\
{ }^{68} \mathrm{Ge}{ }^{\mathrm{CE}} \rightarrow{ }^{68} \mathrm{Ga} \\
{ }^{235} \mathrm{U}(\mathrm{n}, \mathrm{f})^{90} \mathrm{Sr}^{\beta-} \rightarrow{ }^{90} \mathrm{Y} \\
{ }^{187} \mathrm{~W}(\mathrm{n}, \gamma)^{188} \mathrm{~W}^{\beta-} \rightarrow{ }^{188} \mathrm{Re} \\
{ }^{228} \mathrm{Th} \rightarrow \ldots \rightarrow{ }^{224} \mathrm{Ra} \rightarrow \\
{ }^{212} \mathrm{~Pb}^{\beta-} \rightarrow{ }^{212} \mathrm{Bi} \\
{ }^{229} \mathrm{Th} \rightarrow \ldots \rightarrow{ }^{225} \\
\mathrm{Ac}^{\alpha} \rightarrow{ }^{221} \mathrm{Fr}^{\alpha} \rightarrow \\
{ }^{217} \mathrm{At}^{\alpha} \rightarrow{ }^{213} \mathrm{Bi}\end{array}$ \\
\hline
\end{tabular}

NOTA: TI - transição isomérica; CE - captura eletrônica; $\mathrm{f}$ - fissão; $\mathrm{d}$ - dêuteron; $\mathrm{n}$ - neutron; $\mathrm{p}$ - próton.

eluídos isentos do radionuclídeo "pai", do material que constitui a coluna, assim como isento de outros possíveis radionuclídeos contaminantes.

As características dos sistemas de geradores com importância em Medicina Nuclear estão resumidas na Tabela II (Saha, 1998).

\section{RADIONUCLÍDEOS PARA DIAGNÓSTICO OU TERAPIA}

Quando um radiofármaco é administrado a um paciente sofre, de modo geral, processos de distribuição, 
TABELA II - Geradores utilizados em Medicina Nuclear

\begin{tabular}{|c|c|c|c|c|c|c|c|}
\hline $\begin{array}{l}\text { Nuclídeo } \\
\text { "pai" }\end{array}$ & $\begin{array}{c}\mathrm{t}_{1 / 2} \text { nuclídeo } \\
\text { "pai" }\end{array}$ & $\begin{array}{l}\text { Reação } \\
\text { nuclear }\end{array}$ & $\begin{array}{l}\text { Nuclídeo } \\
\text { "fillho" }\end{array}$ & $\begin{array}{l}\mathrm{t}_{1 / 2} \text { nuclídeo } \\
\text { "filho" }\end{array}$ & $\begin{array}{c}\text { Tipo de } \\
\text { decaimento } \\
\text { do nuclídeo "filho" }\end{array}$ & $\begin{array}{c}\text { Energia } \\
(\mathrm{keV})\end{array}$ & Eluente \\
\hline${ }^{99} \mathrm{Mo}$ & $66 \mathrm{~h}$ & Fissão ${ }^{98} \mathrm{Mo}(\mathrm{n}, \mathrm{g})$ & ${ }^{99 \mathrm{~m}} \mathrm{Tc}$ & $6 \mathrm{~h}$ & TI & 140 & $\mathrm{NaCl} 0,9 \%$ \\
\hline${ }^{113} \mathrm{Sn}$ & $115 \mathrm{~d}$ & ${ }^{112} \mathrm{Sn}(\mathrm{n}, \mathrm{g})$ & ${ }^{113 \mathrm{~m}} \mathrm{In}$ & $99,5 \mathrm{~min}$ & TI & 392 & $\mathrm{HCl} 0,05 \mathrm{~N}$ \\
\hline${ }^{87} \mathrm{Y}$ & $80 \mathrm{~h}$ & ${ }^{88} \mathrm{Sr}(\mathrm{p}, 2 \mathrm{n})$ & ${ }^{87 \mathrm{~m}} \mathrm{Sr}$ & $2,8 \mathrm{~h}$ & TI & 388 & $\mathrm{NaHCO}_{3} 0,15 \mathrm{M}$ \\
\hline${ }^{68} \mathrm{Ge}$ & $271 \mathrm{~d}$ & ${ }^{69} \mathrm{Ga}(\mathrm{p}, 2 \mathrm{n})$ & ${ }^{68} \mathrm{Ga}$ & $68 \mathrm{~min}$ & $\mathrm{~b}+$ & 511 & EDTA $0,005 \mathrm{M}$ \\
\hline${ }^{62} \mathrm{Zn}$ & $9,3 \mathrm{~h}$ & ${ }^{63} \mathrm{Cu}(\mathrm{p}, 2 \mathrm{n})$ & ${ }^{62} \mathrm{Cu}$ & $9,7 \mathrm{~min}$ & $\mathrm{~b}+$ & 511 & $\mathrm{HCl} 2 \mathrm{~N}$ \\
\hline${ }^{81} \mathrm{Rb}$ & $4,6 \mathrm{~h}$ & ${ }^{79} \mathrm{Br}(\mathrm{a}, 2 \mathrm{n})$ & ${ }^{81 \mathrm{~m}} \mathrm{Kr}$ & $13 \mathrm{~s}$ & TI & 190 & Água ou ar \\
\hline${ }^{82} \mathrm{Sr}$ & $25,5 \mathrm{~d}$ & ${ }^{85} \mathrm{Rb}(\mathrm{p}, 4 \mathrm{n})$ & ${ }^{82} \mathrm{Rb}$ & $75 s$ & $\mathrm{~b}+$ & 511 & $\mathrm{NaCl} 0,9 \%$ \\
\hline
\end{tabular}

metabolização e excreção como qualquer outro fármaco. A excreção do radiofármaco faz-se por meio dos mecanismos existentes (excreção renal, biliar, ou outro) e segue uma lei exponencial semelhante ao decaimento do radionuclídeo. O tempo necessário para que a quantidade de radiofármaco existente no organismo se reduza à metade chama-se tempo de meia-vida biológica.

Num sistema biológico, o desaparecimento de um radiofármaco deve-se ao decaimento físico do radionuclídeo e à eliminação biológica do radiofármaco. A combinação destes dois parâmetros é designada por tempo de meia-vida efetiva.

Pretende-se um tempo de meia-vida efetiva suficientemente curto para minimizar a exposição do paciente à radiação, mas suficientemente longo para permitir adquirir e processar as imagens.

Os radiofármacos que se destinam ao diagnóstico clínico têm na sua composição um radionuclídeo emissor $\gamma$. Nesta situação é desejável que o radionuclídeo incorporado no radiofármaco não emita partículas $\alpha$ ou $\beta$, uma vez que estas apenas serviriam para aumentar a dose de radiação absorvida pelo paciente.

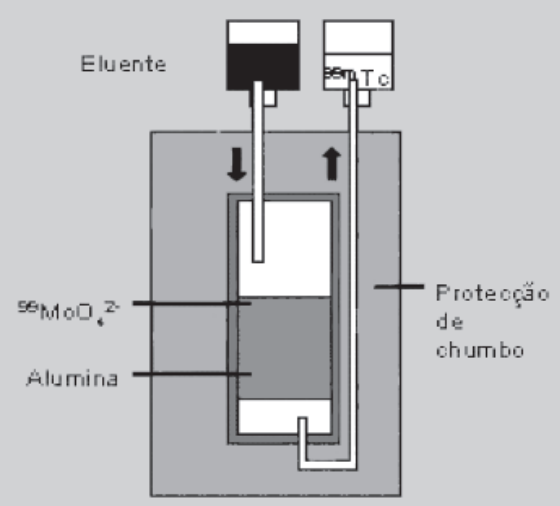

FIGURA 1 - Gerador de ${ }^{99} \mathrm{Mo} /{ }^{99 m} \mathrm{Tc}$.
O radiofármaco deve ser fixado seletivamente pelo órgão ou sistema que se deseja analisar sendo também desejável localização rápida no órgão alvo, metabolização e excreção eficientes, de modo a aumentar o contraste da imagem e reduzir a dose de radiação absorvida pelo paciente.

Um radiofármaco deve ser de fácil produção, baixo custo e facilmente acessível aos Centros de Medicina Nuclear.

A distância geográfica entre o utilizador e o fornecedor limita também a utilização dos radiofármacos contendo radionuclídeos com tempo de meia-vida curto e que não se encontrem disponíveis comercialmente na forma de geradores de radionuclídeos.

\section{RADIOFÁRMACOS PARA DIAGNÓSTICO}

A escolha de um radionuclídeo para o desenvolvimento de um radiofármaco para aplicação em diagnóstico ou terapia em Medicina Nuclear depende principalmente das suas características físicas, nomeadamente o tipo de emissão nuclear, tempo de meia-vida e energia das partículas e/ou radiação eletromagnética emitida.

A energia do fóton $\gamma$ emitido pelo radionuclídeo que entra na composição do radiofármaco para diagnóstico deve situar-se entre os 80-300 keV (Saha, 1998). Isto porque os raios $\gamma$ com energia inferior a $80 \mathrm{keV}$ são absorvidos pelos tecidos e não são detectados exteriormente. Por outro lado, quando a sua energia é superior a $300 \mathrm{keV}$ a eficiência dos detetores atualmente existentes baixa e daí resultam imagens de má qualidade.

Em qualquer dos casos, $o t_{1 / 2}$ deve ser suficiente para preparar o radiofármaco, administrar ao paciente e realizar a imagem.

Na Tabela III estão resumidas as características físicas de radionuclídeos usados em diagnóstico (Saha, 1998; Anderson et al., 1999). 
TABELA III - Radionuclídeos para diagnóstico

\begin{tabular}{lcccc}
\hline Radionuclídeo & Tempo de meia-vida & Modo de decaimento & $\begin{array}{c}\text { Energia raios } \gamma \\
(\mathrm{keV})\end{array}$ & $\begin{array}{c}\text { Abundância da } \\
\text { emissão } \gamma(\%)\end{array}$ \\
\hline${ }^{99 \mathrm{~m}} \mathrm{Tc}$ & $6 \mathrm{~h}$ & $\mathrm{~T} \mathrm{I}$ & 140 & 89 \\
${ }^{131} \mathrm{I}$ & $193 \mathrm{~h}$ & $\beta^{-}, \mathrm{g}$ & 364 & 81 \\
${ }^{123} \mathrm{I}$ & $13 \mathrm{~h}$ & $\mathrm{CE}$ & 159 & 83 \\
${ }^{67} \mathrm{Ga}$ & $78 \mathrm{~h}$ & $\mathrm{CE}$ & $93,185,300,394$ & $37,20,17,5$ \\
${ }^{111} \mathrm{In}$ & $67 \mathrm{~h}$ & $\mathrm{CE}$ & 171,245 & 90,94 \\
${ }^{201} \mathrm{Tl}$ & $73 \mathrm{~h}$ & $\mathrm{CE}$ & 135,167 & 3,20 \\
${ }^{11} \mathrm{C}$ & $20,4 \mathrm{~min}$ & $\beta^{+}$ & 511 & 99,8 \\
${ }^{13} \mathrm{~N}$ & $10 \mathrm{~min}$ & $\beta^{+}$ & 511 & 100 \\
${ }^{15} \mathrm{O}$ & $2,07 \mathrm{~min}$ & $\beta^{+}$ & 511 & 99,9 \\
${ }^{18} \mathrm{~F}$ & $110 \mathrm{~min}$ & $\beta^{+}$ & 511 & 96,9 \\
${ }^{124} \mathrm{I}$ & $4,2 \mathrm{dias}$ & $\beta^{+}$ & 511 & 25 \\
${ }^{64} \mathrm{Cu}$ & $13 \mathrm{~h}$ & $\beta^{+}$ & 511 & 38 \\
\hline
\end{tabular}

NOTA: TI - transição isomérica; CE - captura eletrônica.

Os radiofármacos utilizados para diagnóstico estão classificados em radiofármacos de perfusão (ou $1^{a}$ geração) e radiofármacos específicos (ou $2^{\mathrm{a}}$ geração) (Dilworth et al., 1998). Os radiofármacos de perfusão são transportados no sangue e atingem o órgão alvo na proporção do fluxo sanguíneo. Não têm locais específicos de ligação e pensa-se que são distribuídos de acordo com tamanho e carga do composto. Os radiofármacos específicos são direcionados por moléculas biologicamente ativas, como, por exemplo, anticorpos e peptídeos, que se ligam a receptores celulares ou são transportados para o interior de determinadas células. A capacidade da biomolécula reconhecer os receptores vai determinar a fixação do radiofármaco no tecido pretendido e não deverá ser alterada com a incorporação do radionuclídeo (Jurisson et al., 1993; Fichna et al., 2003).

A maior parte dos radiofármacos em uso clínico corresponde a radiofármacos de perfusão, mas atualmente são os radiofármacos específicos que detêm a atenção da investigação na área da química radiofarmacêutica.

Dos radiofármacos utilizados para diagnóstico, os que contêm na sua composição ${ }^{99 \mathrm{~m}} \mathrm{Tc}$ representam cerca de $90 \%$ da totalidade. Este fato deve-se às características físicas do ${ }^{99 m} \mathrm{Tc}: \mathrm{t}_{1 / 2}$ de $6 \mathrm{~h}$, emissão $\gamma$ com energia adequada ao detetor ( $140 \mathrm{keV})$ e disponível em gerador de baixo custo (Dilworth et al., 1998; Jurisson et al., 1993). O tempo de meia-vida de ${ }^{99 \mathrm{~m}} \mathrm{Tc}$ é suficientemente longo para a preparação dos radiofármacos, administração e aquisição das imagens e suficientemente curto para minimizar a dose de radiação para o paciente.

$\mathrm{Na}$ forma de pertecnetato, tal como é obtido do gerador, o ${ }^{99 \mathrm{~m} T c}$ é quimicamente estável. Contudo, como o ${ }^{99 \mathrm{~m}} \mathrm{Tc}$ é um metal de transição (pertence ao grupo 7 da Tabela periódica) pode existir em 9 estados de oxidação $(-1$ a +7), o que lhe dá a possibilidade de formar complexos de coordenação com numerosos agentes quelantes. A coordenação de agentes quelantes ao ${ }^{99 \mathrm{~m}} \mathrm{Tc}$ é feita quando o metal se encontra em estados de oxidação inferiores ao VII. A redução do metal, do estado de oxidação VII para outros estados de oxidação, é realizada normalmente com cloreto estanoso (Jurisson et al., 1993; Rakias et al., 1996).

Os radiofármacos específicos são classificados de acordo com o receptor específico ou o alvo específico. Os radiofármacos desenvolvidos para se ligarem a receptores têm como objetivo detectar alterações na concentração dos mesmos em tecidos biológicos, especificamente em tecidos tumorais para os quais a expressão dos receptores se encontra alterada significativamente pela diferenciação celular.

São vários os fatores que influenciam na interação dos radiofármacos com os receptores (Vallabhajosula, 2001):

- Depuração plasmática: os compostos para ligação aos receptores (peptídeos, esteróides, neurotransmissores) são de pequeno tamanho e eliminados rapidamente da corrente sanguínea;

- Atividade específica: é necessária elevada atividade específica, uma vez que os receptores apresentam baixa concentração, de modo a evitar a sua saturação com os ligantes "frios";

- Afinidade e Especificidade: o radiofármaco deve ter elevada afinidade para determinado receptor e muito pouca afinidade para os restantes. Este fato é muito importante, pois as concentrações de radiofármaco e receptores são baixas, devendo haver ligação suficientemente forte para a realização da aplicação clínica; 
TABELA IV - Radiofármacos de perfusão para diagnóstico clínico

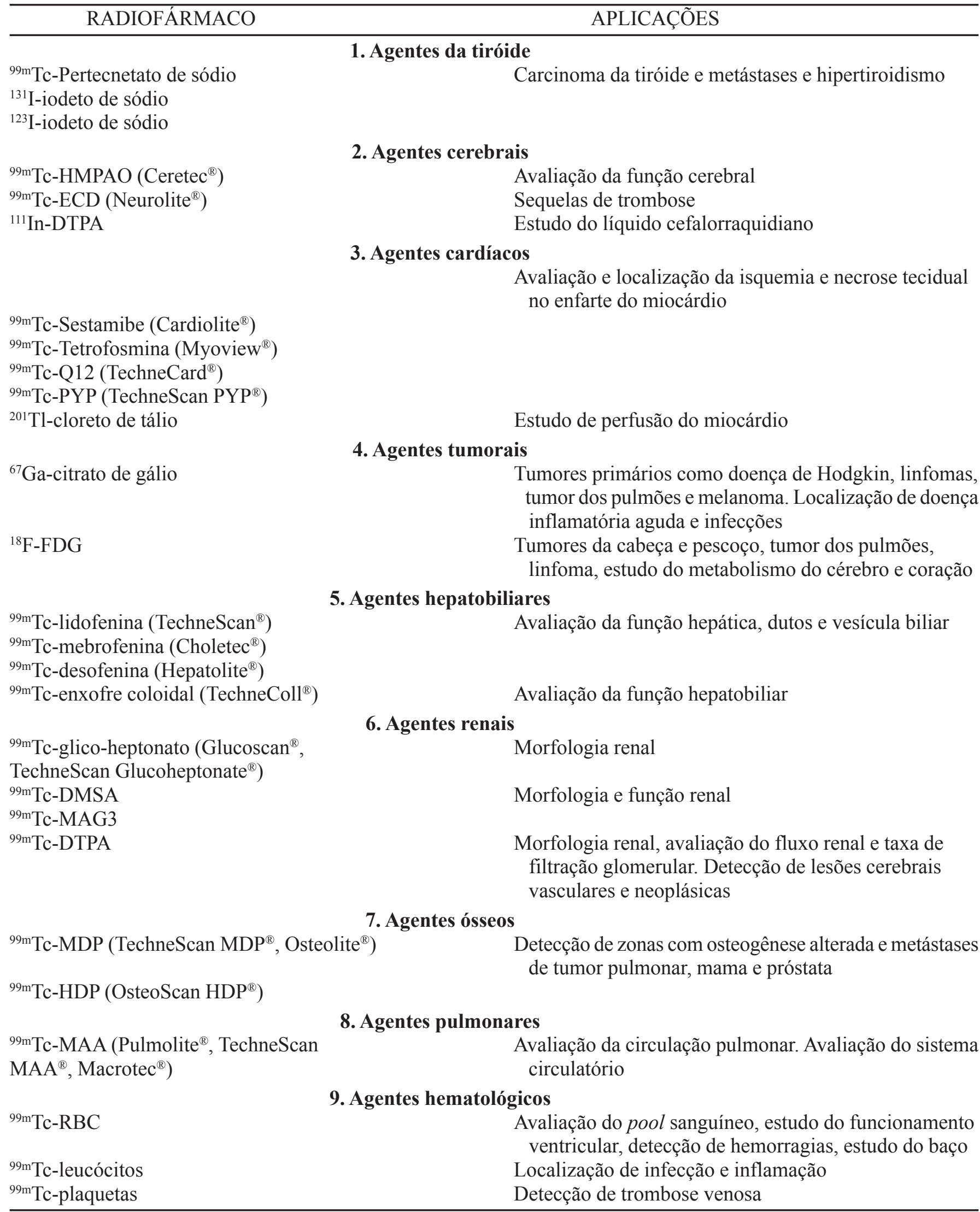


TABELA V - Radiofármacos específicos para diagnóstico clínico

\begin{tabular}{|c|c|}
\hline RADIOFÁRMACO & APLICAÇÕES \\
\hline \multicolumn{2}{|c|}{ 1. Receptores da somatostatina } \\
\hline${ }^{111}$ In-pentetreotida $\left(\right.$ OctreoScan $\left.{ }^{\circledR}\right)$ & $\begin{array}{l}\text { Tumores neuroendócrinos e metástases, como } \\
\text { gastrinoma, neuroblastoma, adenoma da pituitária, } \\
\text { carcinoma medular da tiróide }\end{array}$ \\
\hline${ }^{99 m}$ Tc-P829 $\left(\right.$ NeoTec $\left.^{\circledR}\right)$ & Tumor do pulmão \\
\hline \multicolumn{2}{|c|}{ 2. Receptores do SNC } \\
\hline${ }^{99 m}$ Tc-TRODAT-1 & Doença de Parkinson e esquizofrenia \\
\hline 3. Recepto & $\begin{array}{l}\text { LDL } \\
\text { Estudo das doenças das glândulas adrenais como } \\
\text { adenoma adrenal e doença de Cushing }\end{array}$ \\
\hline 4. Receptores adren & $\begin{array}{l}\text { pré-sinápticos } \\
\text { Tumores neuroendócrinos, feocromocitoma e } \\
\text { neuroblastoma }\end{array}$ \\
\hline \multicolumn{2}{|c|}{ 5. Agentes tromboembólicos } \\
\hline \multicolumn{2}{|c|}{ 6. Anticorpos monoclonais } \\
\hline${ }^{99 \mathrm{~m}} \mathrm{Tc}$-arcitumomabe $\left(\mathrm{CEA}-\mathrm{Scan}^{\circledR}\right)$ & Carcinoma do cólon e reto e metástases \\
\hline${ }^{99 m}$ Tc-nofetumomabe $\left(\right.$ Verluma $\left.^{\circledR}\right)$ & Tumor de pequenas células pulmonares \\
\hline${ }^{111}$ In-satumomabe pendetida (OncoScint CR/OV $\left.{ }^{\circledR}\right)$ & Tumor coloretal e dos ovários \\
\hline${ }^{111}$ In-capromabe pendetida $\left(\right.$ ProstaScint $^{\mathbb{B}}$ ) & $\begin{array}{l}\text { Tumor primário da próstata, metástases e hipertrofia da } \\
\text { próstata }\end{array}$ \\
\hline${ }^{111}$ In-imciromabe pendetida $\left(\right.$ MyoScint $\left.{ }^{\circledR}\right)$ & Detecção de áreas necrosadas no enfarte do miocárdio \\
\hline
\end{tabular}

- Estabilidade in vivo para que o radiofármaco alcance intacto o local alvo;

- Fluxo sanguíneo: a captação do radiofármaco depende do fluxo sanguíneo, perfusão tecidual, permeabilidade capilar e capacidade de difusão.

A Tabela IV apresenta radiofármacos de perfusão usados em diagnóstico e as suas aplicações (Jurisson et al., 1993; Rakias et al., 1996; Dilworth et al., 1998; Saha, 1998; Anderson et al., 1999).

A Tabela $\mathrm{V}$ apresenta radiofármacos específicos usados em diagnóstico e as suas aplicações (Dilworth et al., 1998; Liu et al., 1999; Jurisson et al., 1999; Vallabhajosula, 2001; Johannsen, 2001; Fichna et al., 2003).

\section{RADIOFÁRMACOS PARA TERAPIA}

Os radionuclídeos que emitem partículas ionizantes (partículas $\alpha$, $\beta$, ou elétrons Auger) são indicados para tratamento de tumores. O tipo de partícula a utilizar depende do tamanho do tumor, da distribuição intratumoral e farmacocinética do radiofármaco.

Como já foi referido, a emissão de raios $\gamma$ pode acompanhar a emissão de partículas, mas não contribui em nada para a eficácia da terapia e, pelo contrário, vai aumentar a dose de radiação para os tecidos saudáveis. Mas, sempre que a energia dos raios $\gamma$ é adequada para a aquisição de imagens, pode-se constituir em vantagem, pois permite visualizar a distribuição in vivo do radiofármaco que está sendo utilizado na terapia (Volkert et al., 1999; Heeg et al., 1999).

O tempo de meia-vida é um parâmetro essencial na escolha de radionuclídeos para terapia, devendo adequarse à farmacocinética do radiofármaco e ao tipo de tumor (Jurisson et al., 1993).

\section{Radionuclídeos emissores de partículas $\beta$}

São os radionuclídeos mais utilizados em terapia. As partículas $\beta$ - permitem uma dose de radiação uniforme apesar da sua deposição nos tecidos alvo (tumores) ser heterogênea.

\section{Radionuclídeos emissores de partículas $\alpha$}

Estes radionuclídeos são os escolhidos quando se pretende que a radiação tenha um pequeno alcance. 
Apesar de existirem mais de 100 radionuclídeos emissores $\alpha$, a maioria apresenta tempos de meia-vida demasiado longos, incompatíveis com as aplicações in vivo sendo também de difícil produção. Como resultado, são apenas três os radionuclídeos emissores a cuja aplicação terapêutica está em estudo.

\section{Radionuclídeos emissores de elétrons Auger}

Os elétrons Auger apresentam capacidade ionizante baixa, quando situados no citoplasma das células, mas elevada, quando incorporados em compostos que interagem diretamente com o DNA.

Ainda não existem radiofármacos comercializados emissores de elétrons Auger, mas a concepção de radiofármacos baseados nos elétrons Auger constitui-se em área ativa de investigação. Será necessário conceber um radiofármaco específico internalizado pelas células e que atinja o núcleo das mesmas.

A Tabela VI apresenta os principais radionuclídeos com potencial terapêutico, alguns em utilização e outros em estudo (Volkert et al., 1999; Vallabhajosula, 2001).

Na Tabela VII estão apresentados os radiofármacos para terapia e as suas indicações terapêuticas (Saha, 1998; Volkert et al., 1999; Heeg et al., 1999; Vallabhajosula, 2001).

\section{MÉTODOS DE OBTENÇÃO DE IMAGEM}

Existem essencialmente duas técnicas que permitem a obtenção de imagens em Medicina Nuclear: a técnica de
SPECT (Single Photon Emission Computed Tomography) e a de PET (Positron Emission Tomography).

No primeiro caso, os radiofármacos utilizados têm na sua composição radionuclídeos emissores de radiação $\gamma$ e na segunda técnica utilizam-se radiofármacos que têm na sua composição emissores de pósitrons $\left(\beta^{+}\right)$ (Saha, 1998).

\section{SPECT}

As imagens de SPECT são obtidas utilizando-se câmaras-gama, associadas a computadores que fazem a aquisição e o tratamento de dados, bem como a um sistema que permite visualizar e registrar as imagens.

Sucintamente, a câmara-gama é constituída essencialmente por (Saha, 1998):

- Detetor: é constituído por um ou mais cristais de iodeto de sódio. Da interação da radiação $\gamma$ com estes detetores resulta um sinal luminoso.

- Colimador de chumbo: seleciona a radiação. As câmaras-gama utilizam diferentes tipos de colimadores, concebidos de forma a serem utilizados para uma determinada faixa de energia com resolução e sensibilidade bem determinadas. Limita o campo da radiação, limitando, portanto, a radiação que chega ao detetor. A seleção do colimador depende do tipo de estudo clínico e amplifica o sinal enviado dos tubos fotomultiplicadores.

Esta técnica produz uma imagem tomográfica, que mostra a distribuição da radiação no corpo do paciente, à

TABELA VI - Radionuclídeos para terapia

\begin{tabular}{|c|c|c|c|c|c|}
\hline Radionuclídeo & $\begin{array}{c}\text { Tempo de } \\
\text { meia-vida (dias) }\end{array}$ & $\begin{array}{l}\text { Modo de } \\
\text { decaimento }\end{array}$ & $\begin{array}{c}\text { Energia máxima } \\
\beta(\mathrm{MeV})\end{array}$ & $\begin{array}{c}\text { Energia raios } \\
\gamma(\mathrm{MeV})\end{array}$ & $\begin{array}{l}\text { Alcance máximo } \\
\text { nos tecidos (mm) }\end{array}$ \\
\hline${ }^{131} \mathrm{I}$ & 8,0 & $\beta^{-}$ & 0,81 & $0,364(81 \%)$ & 2,4 \\
\hline${ }^{32} \mathrm{P}$ & 14,3 & $\beta^{-}$ & 1,71 & & 8,7 \\
\hline${ }^{67} \mathrm{Cu}$ & 2,6 & $\beta^{-}$ & 0,57 & $\begin{array}{l}0,185(48 \%) \\
0,092(23 \%)\end{array}$ & - \\
\hline${ }^{177} \mathrm{Lu}$ & 6,7 & $\beta^{-}$ & 0,5 & $\begin{array}{c}0,113(6,4 \%) \\
0,208(11 \%)\end{array}$ & - \\
\hline${ }^{89} \mathrm{Sr}$ & 50,5 & $\beta^{-}$ & 1,46 & & 8,0 \\
\hline${ }^{186} \mathrm{Re}$ & 3,8 & $\beta$ & 1,07 & $0,13 \overline{7}(9 \%)$ & 5,0 \\
\hline${ }^{153} \mathrm{Sm}$ & 1,9 & $\beta$ & 0,8 & $0,103(29 \%)$ & 3,0 \\
\hline${ }^{90} \mathrm{Y}$ & 2,7 & $\beta$ & 2,27 & & 12,0 \\
\hline${ }^{188} \mathrm{Re}$ & 0,71 & $\beta$ & 2,11 & $0,155 \overline{(15 \%)}$ & 10,8 \\
\hline${ }^{117 \mathrm{~m} S n}$ & 13,6 & $\beta$ & 0,13 & $0,158(87 \%)$ & 0,3 \\
\hline${ }^{213} \mathrm{Bi}$ & $0,76 \mathrm{~h}$ & $\alpha$ & 8,0 & $0,440(17 \%)$ & 0,1 \\
\hline${ }^{212} \mathrm{Bi}$ & $1 \mathrm{~h}$ & $\alpha$ & 6,0 & $0,727(7 \%)$ & $70,0 \mathrm{~mm}$ \\
\hline${ }^{211} \mathrm{At}$ & 0,30 & $\alpha$ & 6,0 & $0,670(0,3 \%)$ & $65,0 \mathrm{~mm}$ \\
\hline${ }^{125} \mathrm{I}$ & 60,3 & $\mathrm{CE}$ & $0,4 \mathrm{keV}$ ( $\mathrm{e}^{-}$Auger $)$ & $25-35 \mathrm{keV}$ & $10,0 \mathrm{~nm}$ \\
\hline
\end{tabular}

Nota: CE - captura eletrônica 
TABELA VII - Radiofármacos para terapia

\begin{tabular}{|c|c|}
\hline RADIOFÁRMACO & APLICAÇÕES \\
\hline \multicolumn{2}{|c|}{ 1. Agentes da tiróide } \\
\hline${ }^{131}$ I-iodeto de sódio & $\begin{array}{l}\text { Tratamento do hipertiroidismo e carcinoma papilar e } \\
\text { folicular da tiróide }\end{array}$ \\
\hline \multicolumn{2}{|c|}{ 2. Agentes tumorais de cavidades } \\
\hline${ }^{32} \mathrm{P}$-fosfato de cromo coloidal & $\begin{array}{l}\text { Tratamento de metástases intraperitoneais, como tumor } \\
\text { dos ovários, renal, gastrointestinal }\end{array}$ \\
\hline \multicolumn{2}{|c|}{ 3. Agentes tumorais ósseos } \\
\hline 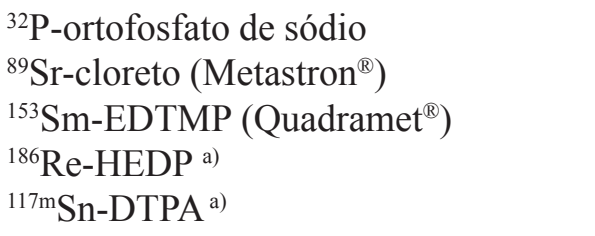 & Tratamento paliativo da dor nas metástases ósseas \\
\hline \multicolumn{2}{|c|}{ 4. Agentes neurotumorais } \\
\hline${ }^{131} \mathrm{I}-\mathrm{MIBG}$ & $\begin{array}{l}\text { Tratamento de tumores neuroendócrinos como o } \\
\text { neuroblastoma ou feocromocitoma }\end{array}$ \\
\hline \multicolumn{2}{|l|}{$\begin{array}{l}{ }^{90} \text { Y-DOTA-Tyr }{ }^{3} \text {-octreotideo a) } \\
{ }^{90} \text { Y-DOTA-lanreotideo }^{\text {a) }}\end{array}$} \\
\hline \multicolumn{2}{|c|}{ 5. Radioimunoterapia } \\
\hline${ }^{131} \mathrm{I}$-anticorpo anti-B1 (BEXAR $\left.{ }^{\circledR}\right)^{\text {a) }}$ & Tratamento do linfoma não-Hodgkin \\
\hline${ }^{90} \mathrm{Y}-\mathrm{MX}$-DTPA-anticorpo anti-B1 (IDE & Tratamento do linfoma não-Hodgkin \\
\hline
\end{tabular}

a) ainda em ensaios clínicos

medida que o detetor vai rodando até $180^{\circ}$ ou $360^{\circ}$ à sua volta. É possível a obtenção de imagens nos diversos planos anatômicos (Elder et al., 1994; Saha, 1998).

\section{PET}

Esta técnica utiliza radionuclídeos emissores de pósitrons (partículas $\beta^{+}$): ${ }^{11} \mathrm{C},{ }^{13} \mathrm{~N},{ }^{15} \mathrm{O},{ }^{18} \mathrm{~F},{ }^{124} \mathrm{I},{ }^{64} \mathrm{Cu}$ ou ${ }^{68} \mathrm{Ga}$, entre outros (Saha, 1998).

Os pósitrons reagem instantaneamente com elétrons, emitindo dois fótons $\gamma$ com energia de $511 \mathrm{keV}$ cada um, na mesma direção, mas com sentidos opostos, que são recolhidos externamente num detetor circular, originando imagens tridimensionais. A detecção simultânea dos raios $\gamma$ que têm sentidos opostos evita a presença de um colimador para limitar o campo de detecção. O sistema PET usa detetores múltiplos distribuídos em círculo, cada um ligado ao que se encontra na posição oposta (Saha, 1998).

A imagem cintilográfica obtida permite conhecer a distribuição do radiofármaco no organismo e quantificar a sua fixação em vários órgãos ou tecidos, permitindo o diagnóstico clínico.

\section{PREPARAÇÃO DE RADIOFÁRMACOS}

Existem alguns fatores muito importantes a serem considerados na preparação de um radiofármaco (Saha, 1998):

- Eficiência do processo de marcação: é desejável um elevado rendimento de marcação, embora nem sempre seja fácil de se obter.

- Estabilidade química do composto: diz respeito à ligação química entre o radionuclídeo e o composto que a ele se coordena ou no qual ele está incorporado.

- Condições físico-químicas da marcação: podem alterar a estrutura ou propriedades biológicas do composto, como por exemplo desnaturação de proteínas por calor excessivo ou valores de $\mathrm{pH}$ extremos.

- Condições de armazenamento: a temperatura e luz podem levar à degradação do composto radioativo, por isso, as condições de armazenamento deverão ser controladas.

- Radiólise: muitos compostos marcados decompõem-se por ação da radiação emitida pelo próprio radionuclídeo e este efeito aumenta quanto maior for a atividade específica do composto. A radiólise pode provocar a quebra da ligação química entre o 
radionuclídeo e a molécula, ou pode interagir com o solvente formando radicais livres, que também podem ter efeito nocivo para o composto radioativo, promovendo o aparecimento de impurezas radioquímicas.

- Prazo de validade: tempo durante o qual o radiofármaco pode ser usado com segurança para o fim a que se destina. A perda de eficácia depende do tempo de semi-desintegração do radionuclídeo, do tipo de solvente, dos excipientes, do tipo de radiação emitida e da natureza da ligação química entre o radionuclídeo e o composto ao qual ele se liga.

\section{Preparações radiofarmacêuticas}

As preparações radiofarmacêuticas são empregadas na prática de Medicina Nuclear em exames de diagnóstico e em terapêutica. Devem, por isso, reunir série de características para assegurar que as doses de radiação que o paciente recebe sejam as mínimas possíveis e o resultado obtido seja o esperado (Rakias et al., 1996).

\section{Preparações radiofarmacêuticas prontas para uso}

São radiofármacos que incorporam na sua estrutura um radionuclídeo com meia-vida suficientemente longa para permitir a sua produção industrial e distribuição, desde o laboratório produtor até ao local de aplicação. São fornecidos na sua forma final, prontos a usar ou exigindo apenas operações simples de diluição ou reconstituição, para preparação de doses individuais de acordo com uma prescrição concreta.

As operações de reconstituição, diluição e fracionamento devem realizar-se em condições higiênicas. As doses preparadas devem ser etiquetadas, indicando a identificação do radiofármaco, atividade, prazo de validade e precauções.

Preparações radiofarmacêuticas preparadas a partir de produtos semi-manufaturados ("kits frios")

A maior parte dos radiofármacos são preparados a partir de produtos semi-manufaturados, pois os radionuclídeos que entram na sua constituição apresentam tempo de meia-vida curto, o que requer normalmente a preparação do radiofármaco imediatamente antes da sua administração.

Um "kit frio" corresponde a formulação contendo o composto a marcar, assim como outros reagentes necessários à reação e também outros excipientes, na forma de liofilizado e em atmosfera inerte de nitrogênio.
Normalmente, a preparação de radiofármacos faz-se por adição de um radionuclídeo, que é obtido a partir de um gerador, originando rapidamente um radiofármaco pronto a ser usado.

A preparação de cada radiofármaco deve realizarse seguindo criteriosamente as instruções fornecidas pelo produtor: atividade a utilizar, condições de marcação, precauções especiais. Deve, ainda, proceder-se à determinação da pureza radioquímica após a preparação do radiofármaco.

Preparações radiofarmacêuticas de ${ }^{99 m}$ TC (Saha, 1998; Rakias et al., 1996):

Os radiofármacos de tecnécio são preparados pela adição de pertecnetato de sódio a um "kit" liofilizado, que contém os componentes necessários para preparar o composto radioativo :

- composto químico a se ligado ao radionuclídeo (responsável pela biodistribuição do radiofármaco após a sua administração);

- o agente redutor para reduzir o pertecnetato $\left(\mathrm{SnCl}_{2}\right)$;

- aditivos e agentes conservantes (agentes antimicrobianos, antioxidantes, estabilizantes, entre outros).

A mistura dos diferentes componentes é fornecida num recipiente adequado, na forma de liofilizado e em atmosfera de nitrogênio, para sua correta conservação.

Tal como estabelece a monografia geral de preparações radiofarmacêuticas da Farmacopéia Européia (2005), após a marcação é necessário etiquetar a preparação, especificando as seguintes informações:

- identificação do radiofármaco;

- nome do preparador;

- atividade total;

- concentração radioativa;

- hora de preparação;

- prazo de validade;

- indicações especiais, se houver.

A preparação radiofarmacêutica conservar-se-á, após a marcação nas condições necessárias, segundo cada caso, durante o prazo de validade da mesma. Este prazo de validade é variável, indo desde os 30 minutos até as 6 h de validade, que normalmente se aceitam para os radiofármacos de tecnécio. Durante este período podem se retirar doses sucessivas dos frascos, cada uma delas adequada para a administração.

Após a marcação e antes da administração do radiofármaco de tecnécio, é necessário realizar os testes adequados de controle de qualidade para comprovar os requisitos impostos para sua administração. 
Preparações radiofarmacêuticas autólogas (Rakias et al., 1996; Balaban et al., 1986):

São radiofármacos resultantes da marcação com radionuclídeos de amostras do paciente (geralmente células sanguíneas), que são depois readministradas. A preparação de radiofármacos a partir de elementos celulares sanguíneos implica o manuseio de amostras de sangue do paciente, requerendo, normalmente, manipulação muito maior do que a preparação de outros radiofármacos sendo um processo aberto. Por este motivo é necessário extremar as medidas sanitárias quando se procede à sua preparação.

A preparação de radiofármacos autólogos, pelo tipo de manipulação que requer e pelo caráter absolutamente individual da preparação, deve se considerar como a preparação de uma fórmula magistral injetável, devendo cumprir os requisitos exigidos a estas preparações pela legislação.

Na preparação de radiofármacos baseados em células sanguíneas podem-se utilizar eritrócitos, plaquetas, leucócitos, marcados com diferentes radionuclídeos em função da finalidade.

A marcação de células sanguíneas pode ser efetuada in vitro ou in vivo, sendo a marcação in vitro a que produz melhores imagens cintilográficas.

\section{Controle de qualidade de preparações radiofarmacêuticas}

O controle de qualidade é um dos pontos essenciais nas Normas de Boas Práticas Radiofarmacêuticas (BPR). Engloba série de medidas e processos para assegurar a qualidade do produto final, antes da sua administração. Dada a sua importância, a garantia de qualidade inclui, entre outras, a validação de técnicas, a calibração de aparelhos e equipamento utilizados no processo de produção (Rakias et al., 1996).

As preparações radiofarmacêuticas podem ser de vários tipos e para cada tipo existirá um processo de controle de qualidade, em função da sua própria natureza.

A principal diferença entre medicamentos não radioativos e preparações radiofarmacêuticas reside no prazo de validade que muitas vezes é de horas ou alguns dias para estes últimos. Devido ao curto tempo de meia-vida dos radionuclídeos, muitos radiofármacos têm que ser produzidos, controlados e administrados num curto espaço de tempo, muitas vezes no mesmo dia. O cumprimento das boas práticas de produção é essencial uma vez que, por vezes, o radiofármaco é administrado sem os resultados dos testes de esterilidade e apirogenicidade (Rakias et al., 1996).

Devido à introdução dos “kits" liofilizados, ao curto tempo de meia-vida de alguns radionuclídeos utilizados, ao uso de geradores e radionuclídeos produzidos em cíclotron, tornou-se necessária a produção dos radiofármacos no local onde vão ser administrados.

Há diferentes aspectos a serem controlados nos radiofármacos (Balaban et al., 1986; Rakias et al., 1996; Saha, 1998):

\section{Ensaios comuns a todos os medicamentos}

Galênicos

- ensaios da forma farmacêutica

Físico-químicos

- características físicas

- pH e força iônica

- isotonicidade para injetáveis

- pureza química

Controle biológico

- esterilidade

- apirogenicidade

\section{Ensaios específicos dos radiofármacos}

Controle de parâmetros específicos relacionados à natureza radioativa do radiofármaco:

- atividade total (quantidade de isótopo radioativo existente no radiofármaco; exprime-se em $\mathrm{Bq}$ )

- atividade específica (atividade por unidade de massa do composto marcado, ou dos isótopos presentes; exprime-se em Bq/mg)

- concentração radioativa (atividade por unidade de volume; exprime-se em $\mathrm{Bq} / \mathrm{mL}$ )

- pureza radionuclídica (porcentagem de atividade do radionuclídeo considerado relativamente à atividade total, que pode ser devida à contaminação por outros radionuclídeos)

- pureza radioquímica (expressa pela percentagem de atividade de um dado radionuclídeo na forma química que se pretende, relativamente à atividade total)

\section{Controle físico-químico}

\section{- Características físicas}

Incluem a observação da cor da preparação e a avaliação da presença de partículas estranhas. Sempre que as preparações são de natureza coloidal ou agregados, tais como ${ }^{99 \mathrm{~m}} \mathrm{Tc}$-enxofre coloidal e ${ }^{99 \mathrm{~m}} \mathrm{Tc}-\mathrm{MAA}$, é particularmente importante a avaliação do tamanho e número das partículas presentes na preparação. O tamanho das partí- 
culas é um fator determinante da biodistribuição do radiofármaco (Saha, 1998).

- pH e força iônica

$\mathrm{O} \mathrm{pH}$ é muito importante para a estabilidade da preparação e deverá estar próximo de 7,4 (pH sanguíneo). Contudo, o sangue apresenta alguma capacidade tamponante podendo alargar-se a faixa de $\mathrm{pH}$, se necessário. $\mathrm{O} \mathrm{pH}$ da preparação é normalmente medido com papel indicador, o que evita a exposição do experimentador à radiação e a contaminação do material de medida.

Uma vez que a maioria dos radiofármacos se apresenta na forma de injetáveis, é necessária a avaliação da força iônica, isotonicidade e osmolalidade (Saha, 1998).

- Pureza química

A pureza química é a fração de material na forma química desejada, esteja ou não marcada. As impurezas químicas têm origem na degradação do produto ou adição inadvertida antes, durante ou após a marcação, como por exemplo a presença de alumínio no eluído do gerador de ${ }^{99 \mathrm{~m}} \mathrm{Tc}$.

\section{Controle radioquímico}

- Pureza radionuclídica

A presença de eventuais impurezas radionuclídicas está relacionada com o modo de produção do radionuclídeo ou com uma preparação inadequada dos geradores. A presença de ${ }^{99}$ Mo no eluído de ${ }^{99 m}$ Tc é um exemplo de impureza radionuclídica.

Estas impurezas radionuclídicas aumentam a dose de radiação para o paciente e podem interferir na qualidade das imagens. Podem ser removidas por métodos químicos.

A pureza radionuclídica pode ser determinada pela determinação da energia e tipo de radiações emitidas pelo radiofármaco. Esta avaliação faz-se por espectrometria $\gamma$. Este método não permite identificar os emissores $\beta$, que podem ser determinados por espectrometria $\beta$ ou cintilação líquida (Farmacopéia Portuguesa, 1997; Saha, 1998).

- Pureza radioquímica

As impurezas radioquímicas têm origem na decomposição do radiofármacos, devido à ação do solvente, temperatura, $\mathrm{pH}, \mathrm{luz}$, presença de agentes oxidantes ou redutores, radiólise. No caso dos radiofármacos de ${ }^{99 \mathrm{~m}} \mathrm{Tc}$, as impurezas radioquímicas mais comuns são ${ }^{99 \mathrm{~m}} \mathrm{TcO}_{4}{ }^{-}$ou formas hidrolisadas de ${ }^{99 \mathrm{~m}} \mathrm{Tc}$. Estas impurezas podem au- mentar a dose de radiação e interferir nas imagens de diagnóstico.

A presença de impurezas radioquímicas nas preparações radiofarmacêuticas diminui a qualidade da imagem e aumenta a dose de radiação para o paciente.

As impurezas radioquímicas são determinadas por métodos analíticos, tais como cromatografia em papel, camada delgada, gel, troca iônica, electroforese ou extração com solventes e CLAE (Cromatografia Líquida de Alta Eficiência) (Farmacopéia Européia, 2005; Saha, 1998).

A Farmacopéia Européia estipula que mais de $95 \%$ da atividade deverá corresponder à forma química desejada.

- Concentração radioativa

Este parâmetro determina-se com o auxílio de um medidor de atividade, devidamente calibrado, e conhecendo-se o volume total da amostra (Saha, 1998).

\section{Controle biológico}

- Esterilidade

A esterilidade indica a ausência de microorganismos viáveis na preparação. Todas as preparações injetáveis devem ser esterilizadas por métodos adequados, que dependem da natureza do produto, do solvente e excipientes.

Os métodos de esterilização mais usados são a esterilização por calor úmido, que deve ser aplicado apenas a compostos termoestáveis, e a esterilização por membrana filtrante que é mais adequado a preparações radiofarmacêuticas, uma vez que os volumes manuseados são pequenos, o método é rápido (e por vezes a meia-vida do produto é muito curta) e é aplicável a produtos termolábeis (Rakias et al., 1996).

O ensaio de esterilidade realizado por incubação de amostras de produto em meios de cultura, deve estar de acordo com a Farmacopéia Européia (2005).

- Apirogenicidade

É também um requisito dos injetáveis que sejam livres de pirogênios (Saha, 1998; Rakias et al., 1996; Balaban et al., 1986). Os pirogênios são produtos do metabolismo das bactérias (endotoxinas), com tamanho entre 0,05 e $1 \mathrm{~mm}$, solúveis, termoestáveis e são capazes de induzir hipertermia.

A esterilidade de uma solução não garante apirogenicidade, assim como a esterilização não destrói os pirogênios presentes, por isso, para se preparar um radiofármaco sem pirogênios deverá utilizar-se o método asséptico. 
O ensaio de pirogênios consiste em medir a elevação da temperatura provocada no coelho pela injeção intravenosa de uma solução estéril da preparação a examinar e deverá ser realizado de acordo com a Farmacopéia Européia (2005).

Contudo, é também utilizado outro teste, que avalia mais rapidamente a pirogenicidade das preparações, é o teste L.A.L. (Limulus amoebocytes lysate). Este teste consiste em medir o nível de endotoxinas presentes na preparação utilizando um lisado de amebócitos do Limulus polyphemus. As endotoxinas, quando entram em contacto com o lisado, provocam turvação, precipitação ou gelificação da solução em aproximadamente uma hora. $\mathrm{O}$ teste L.A.L. é proposto pela Farmacopéia Européia (2005), que indica os valores limite na monografia de cada radiofármaco.

A maioria dos países adota as diretrizes de produção dos produtos farmacêuticos, que contêm suplementos relativos aos radiofármacos com indicações de proteção contra radiação e permissão para liberação dos produtos antes de todos os testes realizados (Rakias et al., 1996).

\section{DESENVOLVIMENTO DE NOVOS RADIOFÁRMACOS}

Os radiofármacos comerciais correspondem aos requisitos necessários para o seu uso, mas o que se pretende atualmente são radiofármacos específicos que permitam o diagnóstico precoce de várias patologias ou a terapia extremamente seletiva do órgão alvo. Existe também o problema dos radionuclídeos de tempo de meia-vida curto (PET), que não estão disponíveis a qualquer serviço de Medicina Nuclear, sendo também uma vantagem a sua substituição por radionuclídeos adequados a SPECT. Alguns dos fatores que influenciam a concepção de novos radiofármacos são (Saha, 1998):

- Compatibilidade entre o radionuclídeo e a molécula que a este se pretende ligar, avaliada através do conhecimento das propriedades químicas dos dois componentes;

- Estequiometria, que indica a quantidade a adicionar de cada componente, e é muito importante, principalmente quando se trabalha com concentrações muito baixas. Concentrações demasiado altas ou baixas de algum componente podem afetar a integridade da preparação;

- Carga e tamanho da molécula, que podem determinar a absorção no sistema biológico. Por exemplo, moléculas com massa molecular maior do que 60.000 não são filtradas no glomérulo renal;

- Ligação às proteínas, que afeta a distribuição e depu- ração do radiofármaco, e é influenciada pela carga da molécula, $\mathrm{pH}$, tipo de proteína e concentração de ânions no plasma. As principais proteínas plasmáticas ligantes são albumina, lipoproteínas e transferrina. Em $\mathrm{pH}$ baixo, as proteínas estão carregadas positivamente aumentando a ligação de fármacos aniônicos. Em pH elevado, as proteínas estão carregadas negativamente favorecendo a ligação dos fármacos catiônicos. Um fenômeno que pode ocorrer com os complexos de metais de transição é a transquelatação, que é a troca do íon metálico dos quelatos por uma proteína do plasma, levando à quebra do composto radioativo;

- Solubilidade, que determina a distribuição e localização. Substâncias lipossolúveis difundem-se melhor na membrana celular e, conseqüentemente, maior será a sua localização no órgão alvo. A ligação às proteínas reduz a lipofilia e as moléculas iônicas são menos lipossolúveis do que as moléculas neutras;

- Estabilidade dos radiofármacos, que compromete a sua utilização. Os compostos devem ser estáveis in vitro e in vivo. Devem ser estabelecidas as condições ótimas de temperatura, $\mathrm{pH}$ e luz, pois vão determinar as condições de preparação e armazenamento. A quebra do composto in vivo origina biodistribuição indesejável da radioatividade;

- Biodistribuição, que indica a utilidade e eficácia do radiofármaco. Os estudos de biodistribuição incluem a avaliação da distribuição nos tecidos, a depuração plasmática e o tipo de excreção após administração do radiofármaco. A distribuição tecidual indica se o composto tem interesse para o diagnóstico de determinado órgão e a excreção avalia o tempo durante o qual o paciente vai estar exposto à dose de radiação.

O desenvolvimento de novos radiofármacos para terapia baseia-se na tentativa de aumentar cada vez mais a especificidade pelos locais-alvo, mesmo que esses locais sejam desconhecidos, diminuindo ao máximo a toxicidade para os tecidos saudáveis. Deverão apresentar as seguintes caraterísticas (Volkert et al., 1999):

- Direcionamento seletivo in vivo para as células cancerígenas;

- Capacidade para alcançar elevadas concentrações radioativas e distribuição no tecido tumoral;

- Capacidade para retenção no tecido alvo;

- Capacidade de eliminação dos tecidos saudáveis com o objetivo de minimizar os efeitos secundários. O desenvolvimento de novos radiofármacos é um esforço multidisciplinar, que requer a colaboração de áreas variadas como química, física, biologia e medicina, para 
o melhoramento e obtenção de radiofármacos cada vez mais próximos do ideal.

\section{SÍMBOLOS E ABREVIATURAS}

\begin{tabular}{|c|c|}
\hline$\alpha$ & - partícula alfa \\
\hline$\beta$ & - partícula beta \\
\hline$\beta^{+}$ & - pósitron \\
\hline$v$ & - raios gama \\
\hline BPR & - boas práticas radiofarmacêuticas \\
\hline $\mathrm{Bq}$ & - Becquerel \\
\hline $\mathrm{CE}$ & - captura eletrônica \\
\hline $\mathrm{Ci}$ & - Curie \\
\hline & - dêuteron \\
\hline DMSA & - ácido dimercaptossuccínico \\
\hline DNA & - ácido desoxirribonucléico \\
\hline DOTA & $\begin{array}{l}\text { - ácido 1,4,7,10-tetrazaciclododecano- } \\
\text { N, } N^{\prime}, N^{\prime \prime}, N^{\prime, "} \text {-tetracético }\end{array}$ \\
\hline DTPA & - ácido dietilenotriaminopentacético \\
\hline ECD & - dímero de etilcisteína \\
\hline EDTA & - ácido etilenodiaminotetracético \\
\hline EDTMP & - etilenodiaminotetrametilenofosfonato \\
\hline $\mathrm{eV}$ & - elétron-volt \\
\hline f & - fissão \\
\hline FDG & -2-fluor-2-desoxi-D-glicose \\
\hline HDP & - hidroximetilenodifosfonato \\
\hline HEDP & - hidroxietilenodifosfonato \\
\hline HMPAO & $\begin{array}{l}\text { - hexametilpropilenamina oxima }(3,6,6,9- \\
\text { tetrametil-4,8-diazaundecano-2,10- } \\
\text { dionadioxima) }\end{array}$ \\
\hline L.A.L & - lisado de amebócitos de Limulus \\
\hline MAA & - macroagregados de albumina \\
\hline $\mathrm{MAG}_{3}$ & - mercaptoacetiltriglicina \\
\hline MDP & - metilenodifosfonato \\
\hline MIBG & - meta-iodobenzilguanidina \\
\hline $\mathrm{n}$ & - neutron \\
\hline NP-59 & -6ß-iodometil-19-nor-colesterol \\
\hline $\mathrm{p}$ & - próton \\
\hline PET & - tomografia de emissão de pósitrons \\
\hline $\mathrm{RBC}$ & - glóbulos vermelhos \\
\hline RMN & - ressonância magnética nuclear \\
\hline SPECT & $\begin{array}{l}\text { - tomografia computorizada de emissão de } \\
\text { fóton único }\end{array}$ \\
\hline 11 & - transição isomérica \\
\hline
\end{tabular}

ABSTRACT

\section{Radiopharmaceuticals and applications}

Radiopharmaceuticals are substances without pharmacological activity that are used in Nuclear Medicine for diagnosis and therapy for several diseases. Diagnosis radiopharmaceuticals generally emit $\gamma$ radiation or positrons $\left(\beta^{+}\right)$, because their decay originates penetrating electromagnetic radiation that can cross the tissues and be externally detected. Therapeutic radiopharmaceuticals must include in their composition ionized particles emission nucleus ( $\alpha, \beta$-or Auger electrons), since their action is based in selective tissue destruction. There are two main methods for image acquisition: SPECT (Single Photon Emission Computerized Tomography) that uses $\gamma$ emission radionuclides $\left({ }^{99 m} \mathrm{Tc},{ }^{123} \mathrm{I},{ }^{67} \mathrm{Ga},{ }^{201} \mathrm{Tl}\right)$ and PET (Positron Emission Tomography) that uses positron emission radionuclides like ${ }^{11} \mathrm{C},{ }^{13} \mathrm{~N},{ }^{15} \mathrm{O},{ }^{18} \mathrm{~F}$. Radiopharmaceuticals can be classified into perfusion radiopharmaceuticals (first generation) or specific radiopharmaceuticals (second generation). Perfusion radiopharmaceuticals are transported in the blood e reach the target organ in the direct proportion of the blood stream. Specific radiopharmaceuticals contain a biologically active molecule that binds to cellular receptors that must remain biospecific after binding to the radiopharmaceutical. For this type of radiopharmaceuticals, tissue or organ uptake is determined by the biomolecule capacity of recognizing receptors in those biological structures. Radiopharmaceuticals are produced ready to use, in cold kits or in autologal preparations. According to the preparation type there is a different quality control procedure. Most of the radiopharmaceuticals used nowadays are of the perfusion type. Research focus in the development of specific radiopharmaceuticals that can provide information, at the molecular level, of biochemical alterations associated to different pathologies.

UNITERMOS: Radiopharmaceuticals. Radionuclide. Diagnosis. Therapy.

\section{REFERÊNCIAS BIBLIOGRÁFICAS}

ANDERSON, C.J.; WELCH, M.J. Radiometal-labeled agents (non-technecium) for diagnostic imaging. Chem. Rev., v. 99, p. 2219-2234, 1999.

BALABAN, A.T. Labeled compounds and radiopharmaceuticals aplied in nuclear medicine. John Wiley and Sons Ltd.; 1986.

CLARKE, M.J.; SADLER, P.J. Metallopharmaceuticals IIDiagnosis and therapy. New York: Springer, 1999. p. 48-49. 
DILWORTH, J.R.; PARROT, S.J. The biomedical chemistry of thecnetium and rhenium. Chem. Soc. Rev., v. 27, p. 4355,1998

ELDER, R.C.; TEPPERMAN, K. Metal-based drugs and imaging agents in Encyclopedia of Inorganic Chemistry, King, R. B. (Eds.). New York: John Wiley and Sons, 1994. p. 2165-2176.

EUROPEAN PHARMACOPEIA 5th Ed, Council of Europe. Strasbourg: EDQM, 2005.p. 578

FICHNA, J.; JANECKA, A. Synthesis of target-specific radiolabeled peptides for diagnostic imaging. Bioconjugate Chem., v. 14, p. 1-13, 2003.

GUO, Z.; SADLER, P.J. Metals in Medicine. Angew. Chem. Int. Ed., v. 38, p. 1512-1531, 1999.

HEEG, M.J.; JURISSON, S.S. The role of inorganic chemistry in the development of radiometal agents for cancer therapy. Ass. Chem. Res., v. 32, p.1053-1060, 1999.

JOHANNSEN, B. Tc-99m chemistry and new Tc-99m radiopharmaceuticals. Institute of Bioinorganic and Radiopharmaceutical Chemistry. Rossendorf: Wissenschaftlich-Technische Berichte, 2002. (Annual Report, 2001).

JURISSON, S.; BERNING, D.; JIA, W.; MA, D. Coordination compounds in nuclear medicine. Chem. Rev., v. 93, p. 1137-1156, 1993.
JURISSON, S.S.; LYDON, J.D. Potencial technecium small molecule, radiopharmaceuticals. Chem. Rev., v. 99, p. 2205-2218, 1999.

LIU, S.; EDWARDS, D.S. 99mTc-labeled small peptides as diagnostic radiopharmaceuticals. Chem. Rev., v. 99, p. 2235-2268, 1999.

RAKIAS, F.; ZOLLE, I. Handbook of quality control methods of $99 \mathrm{mTc}$-radiopharmaceuticals in Nuclear Medicine; COST B3: WG-1; 1996.

SAHA, G. B. Fundamentals of nuclear pharmacy. Springer, 1998. p. 34-170.

VALLABHAJOSULA, S. Radiopharmaceuticals in oncology. In: KHALKHALI, I.; MAUBLANT, J.C.; GOLDSMITH, S.J., (Eds.). Nuclear oncology diagnosis and therapy. Philadelphia: Lippincott Williams and Williams, 2001. p. 31-59.

VOLKERT, W.A.; HOFFMAN, T.J. Therapeutic Radiopharmaceuticals. Chem. Rev., v. 99, p. 2269-2292, 1999.

Recebido para publicação em 28 de março de 2005. Aceito para publicação em 30 de janeiro de 2006. 